\title{
High Energy Astrophysical Neutrino Flux Measurement Using Neutrino-induced Cascades Observed in 4 Years of IceCube Data
}

\author{
The IceCube Collaboration ${ }^{\dagger}$ \\ $\dagger$ http://icecube.wisc.edu/collaboration/authors/icrc17_icecube \\ E-mail: hans.niederhausenestonybrook.edu, yiqian.xu@stonybrook.edu
}

\begin{abstract}
We report a new measurement of the diffuse flux of high energy extraterrestrial neutrinos from the entire sky with energies of $\mathrm{O}(1 \mathrm{TeV})$ and above. We have analyzed four years of IceCube data recorded from 2012-2015 focusing on neutrino-induced particle showers. These cascades stem predominantly from electron and tau neutrino interactions, provide good deposited energy resolution and have a lower rate of atmospheric production than muon neutrinos. A new event selection has been developed combining traditional straight cuts with gradient boosted multi-class decision trees to isolate cascades more efficiently, resulting in the largest cascade sample obtained by IceCube to date. The observed astrophysical component dominates at energies above $20 \mathrm{TeV}$ and is well described by a single, unbroken power-law. The preliminary fit result is a spectral index of $\gamma=2.48 \pm 0.08$ with a per-flavor normalization at $100 \mathrm{TeV}$ of $\phi=\left(1.57_{-0.22}^{+0.23}\right) \cdot 10^{-18} \mathrm{GeV}^{-1} \mathrm{~s}^{-1} \mathrm{sr}^{-1} \mathrm{~cm}^{-2}$ in agreement with previous IceCube measurements. We investigated the possibility of a spectral hardening at the upper end of the spectrum by allowing a second power-law component to enter our flux model. No evidence for such hardening has been found. In the near future we expect improved results by adding IceCube's existing cascades from the preceding two years (2010-2011) into this analysis thereby enlarging the total live time to six years.
\end{abstract}

Corresponding authors: Hans Niederhausen*1, Yiqian $\mathrm{Xu}^{1}$

${ }^{1}$ Department of Physics and Astronomy, Stony Brook University, Stony Brook, NY 11794-3800

35th International Cosmic Ray Conference - ICRC2017

10-20 July, 2017

Bexco, Busan, Korea

${ }^{*}$ Speaker. 
1. Introduction ice at the geographic South Pole between depths of $1450 \mathrm{~m}$ and $2450 \mathrm{~m}$. IceCube observes neutrinos based on optical measurements of Cherenkov radiation emitted by secondary particles produced in neutrino interactions. Those interaction are dominated by deep-inelastic scattering (DIS) between neutrinos and nucleons in the ice. Events can be distinguished by the pattern of their light deposition mainly into tracks and cascades. Tracks arise primarily from through-going or starting muons while cascades can be produced by charged-current interactions of $v_{e}$ and $v_{\tau}$ and by neutral current interactions of any flavor. Most particles triggering IceCube are atmospheric muons produced in cosmic ray induced air showers. The same air showers also generate atmospheric neutrinos. In addition IceCube reported the discovery of high energy neutrinos of extraterrestrial origin [2][3]. Their production is assumed to be associated with particle acceleration at cosmic ray sources. Based on diffusive shock acceleration and neutrino oscillations during propagation one expects the flux to exhibit a power-law spectrum $\mathrm{E}^{-\gamma}$ [4][5] with an approximately equal flavor admixture at earth $\left(v_{e}: v_{\mu}: v_{\tau} \approx 1: 1: 1\right)[6]$. Several IceCube analyses have measured the spectrum of astrophysical neutrinos [3][7][8][9]. The observed spectral indices range from $\gamma \sim 2.2$ when muon neutrinos from the Northern Sky are considered, observing astrophysical neutrinos above $119 \mathrm{TeV}$, to $\gamma \sim 2.7$ when cascades are studied with sensitivity primarily to electron and tau neutrinos from the entire sky in the several $\mathrm{O}(10 \mathrm{TeV})$ energy range. Compared to the track channel, the cascade channel benefits from smaller atmospheric background levels as well as from a superior (deposited) energy resolution of $\sim 15 \%(\mathrm{E}>10 \mathrm{TeV})$. We present new results concerning the spectral behavior of the astrophysical neutrino flux using cascade events observed in four years of IceCube data recorded from 2012-2015. In the future this dataset will be combined with the cascades presented in the previous analysis [9], subsequently referred to as "2yr-cascades".

\section{Event Selection}

The event selection we present in this paper improves over the $2 \mathrm{yr}-$ cascade analysis by enhancing the cascade signal efficiency ( $>20 \%$ for reconstructed energies $\left.E_{\text {rec }}>60 \mathrm{TeV}\right)$ and lowering the energy threshold from $\mathrm{E}_{\mathrm{rec}}=10 \mathrm{TeV}$ down to $\mathrm{O}(1 \mathrm{TeV})$ thus reducing systematic uncertainties. The selection criteria were determined by comparing MonteCarlo simulations for signal and background contributions. The simulations were generated using the same software packages as in the previous work [9], most notably full air shower simulation (CORSIKA) to model cosmic-ray induced muon background. The events that are most difficult to identify as background are single muons that deposit most of their energy in a single cascade like energy loss, thus mimicking a cascade like signature. Following ref. [8] we now rely on a parametrization of the single muon yield derived from CORSIKA [10] to more efficiently predict this background. While the modeling of the conventional atmospheric neutrino component remains unchanged [9] (HKKMS06 [11] with modifications [12]) we altered the prediction for prompt atmospheric neutrinos [13] following the updated calculation described in [14] by the same authors. At high energies $\left(\mathrm{E}_{\mathrm{rec}} \geq 60 \mathrm{TeV}\right)$ we isolate cascade events using straight cuts based on the topology variables described in the previous analysis [9][15] or slight modifications thereof. Cut values were chosen to maximize signal efficiency while suppressing the atmospheric muon background expectation to zero. At lower energies a gradient boosted multi-class decision trees classifier [16] is used to suppress atmospheric muon background and separate the remaining events according to their topology into cascades and starting tracks with the latter being dominated by conventional 
atmospheric neutrinos. Each event is assigned three scores: muon score, hybrid score and cascade score measuring the compatibility of the event with a muon, starting track or cascade topology, respectively. Figure 1 (left) shows for one year the the deposited energy distribution of events passing all selection criteria of the high energy selection except the final $\mathrm{E}_{\mathrm{rec}}>60 \mathrm{TeV}$ cut for one year of data. Also shown is the BDT's cascade score distribution used to define the cascade signal region (center). The simulations agree well with the observed data. The final low (high) energy selection achieves a neutrino purity of $>90 \%$ (100\%). Figure 1 (right) shows the effective areas of the cascade sample for the three different neutrino flavors after combining the low and high energy selections and demonstrates sensitivity primarily to electron and tau flavors. In total 19 events were found with reconstructed energies above $100 \mathrm{TeV}$. The highest energy cascade previously not reported by IceCube, is located at a depth with shorter than average absorption length due to high concentration of dust particles in the ice and has been reconstructed with an energy deposit of $2 \mathrm{PeV}$. Further studies using improved estimates of the absorption length at these depths resulted in a lower, more realistic reconstructed energy of $800 \mathrm{TeV}$. The corresponding systematic uncertainties are still under evaluation.
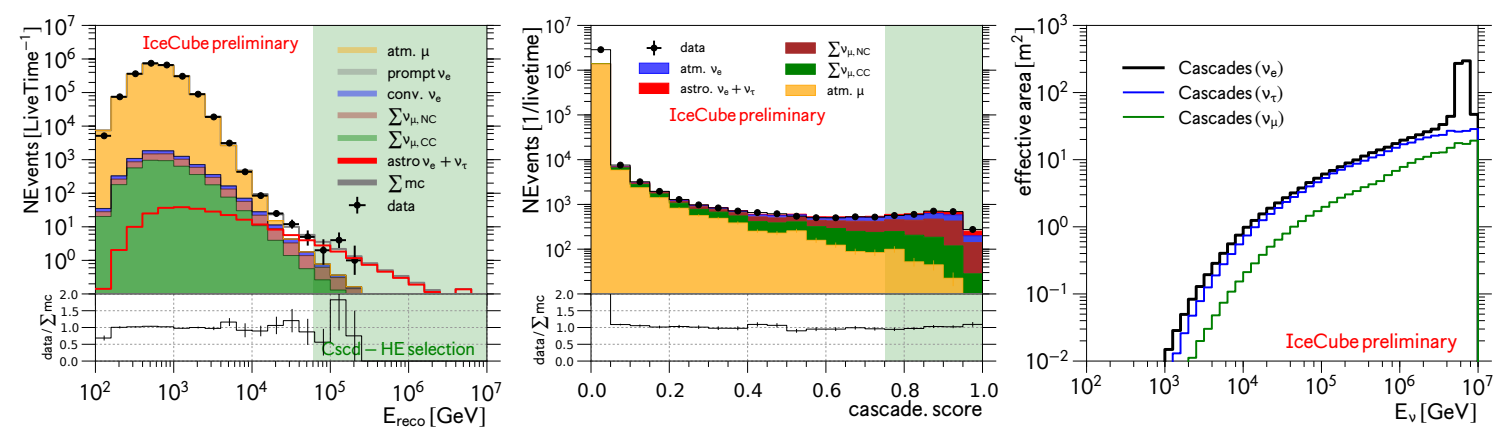

Figure 1: Deposited energy of high energy selection before final energy cut for the year 2015 (left); Distribution of cascade.score (BDT variable) used in low energy selection (center). Signal regions are marked as green shaded bands. Effective areas of the combined cascade samples (low energy $\cup$ high energy) (right).

3. Analysis Method formed by matching the reconstructed energy distribution to the simulation prediction by numerically maximizing a binned poisson likelihood. Similarly, approximate $100(1-\alpha) \%$ confidence regions are obtained using the profile-likelihood method in conjunction with Wilks' theorem, as in ref. [9]. If prior information about the parameters of interest is available, we use a Bayesian method and calculate $100(1-\alpha) \%$ highest posterior density (HPD) credible regions $C$ [17] defined by

$$
\begin{array}{r}
C=\{\theta \in \Theta: \pi(\theta \mid x) \geq k(\alpha)\} \\
1-\alpha \leq P(C \mid x)=\int_{C} \pi(\theta \mid x) \mathrm{d} \theta=\int_{C} f(x \mid \theta) \cdot \pi(\theta) \mathrm{d} \theta
\end{array}
$$

where $k(\alpha)$ is the largest constant satisfying Eq. (2). $\pi(\theta), \pi(\theta \mid x)$ and $f(x \mid \theta) \propto L(\theta \mid x)$ are prior distribution, posterior distribution and likelihood function respectively. The same formalism allows us to check predictions from previous IceCube measurements for any observable quantity $z$ using 

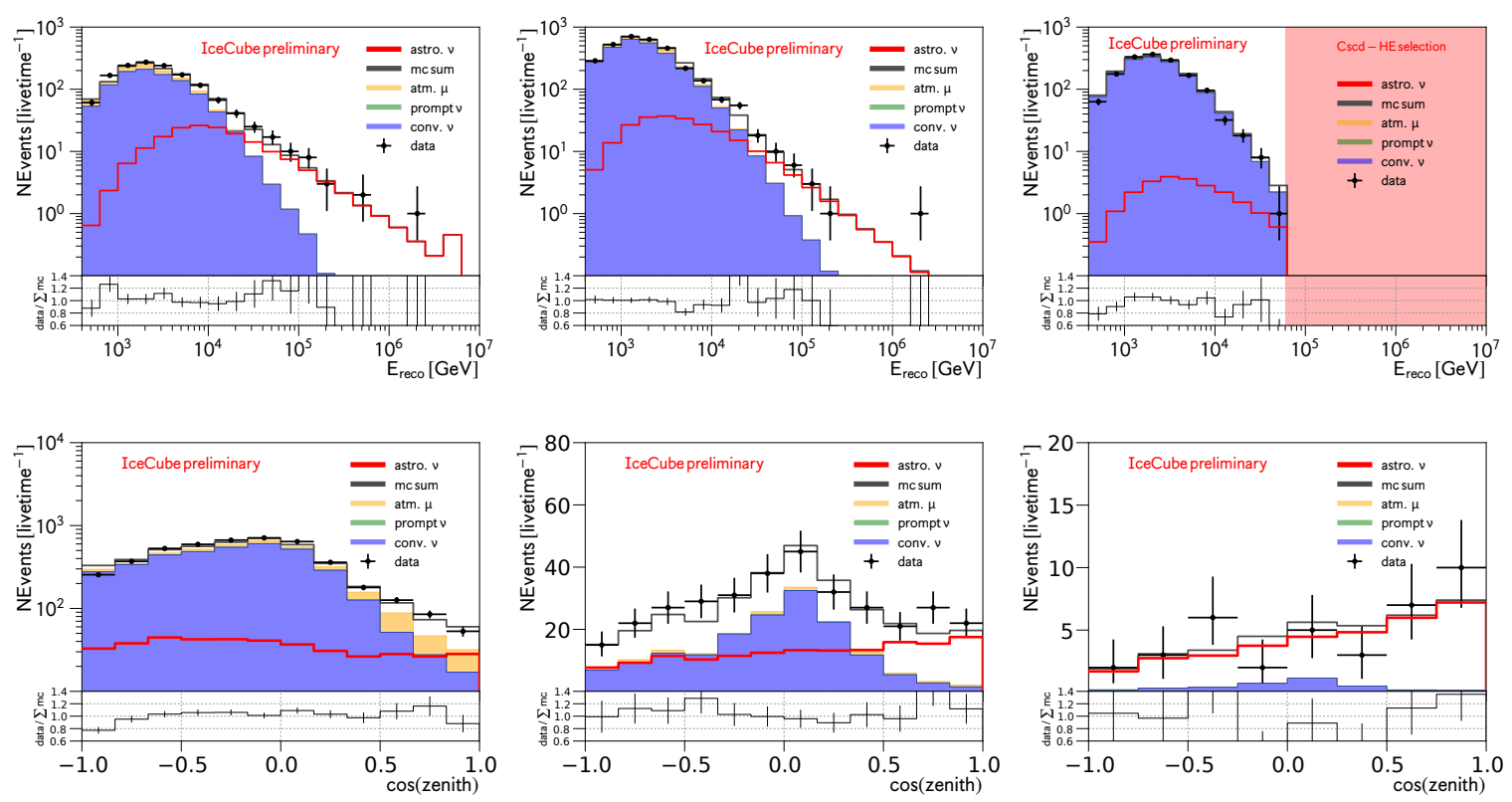

Figure 2: Best fit distributions assuming single power-law model. Top: Energy distributions for cascades (left: Southern sky, center: Northern sky) and starting tracks (right: entire sky). The starting track sample only covers energies up to $\mathrm{E}=60 \mathrm{TeV}$ (red band). Bottom: Cascade zenith distributions for all energies (left), $\mathrm{E}>10 \mathrm{TeV}$ (center) and $\mathrm{E}>60 \mathrm{TeV}$ (right).

predictive densities $p(z \mid y)$ [17], where $y$ refers to data observed in the previous measurement:

$$
p(z \mid y)=\int_{\Theta} g(z \mid \theta) \pi(\theta \mid y) \mathrm{d} \theta .
$$

Tail area probabilities are then defined as $P\left(z \geq z_{o b s} \mid y\right)$ [18], where $z_{o b s}$ is the observed value of $z$ in this sample and can be obtained from the predictive density $p(z \mid y)$ by integration.

We separate the cascade events according to their reconstructed zenith angle $\Theta_{\text {rec }}$ into two groups: 'Northern Sky' $\left(\cos \Theta_{r e c}<0\right)$ and 'Southern Sky' $\left(\cos \Theta_{r e c} \geq 0\right)$. Neutrinos classified as starting tracks from the whole sky form the third group, a $v_{\mu}$-CC control group $\left(\mathrm{E}_{\mathrm{rec}}<60 \mathrm{TeV}\right)$. Finally, we require the total sum of predicted events below the cuts $\left(\mathrm{E}_{\mathrm{rec}}<60 \mathrm{TeV}\right)$ to match the total number of observed events in that region (down to cascade.score=0.1, see Fig. 1) within uncertainties. Sources of systematic uncertainties remain the same as before and are discussed in more detail in ref. [9]. We revised the treatment and implementation of detector related systematic uncertainties (scattering and absorption of photons in the ice, optical efficiency of the DOMs to photons) into our likelihood function to describe the sensitivity of this analysis to these effects at lower energies $\left(\mathrm{E}_{\mathrm{rec}}<10 \mathrm{TeV}\right)$. Each effect is now treated separately and contributes a nuisance parameter to the fit that adjusts the nominal simulation prediction in each bin by independent, multiplicative efficiency corrections. The relationship between the size of the effect, i.e. the value of the nuisance parameter, and the efficiency correction is treated as linear and has been determined from dedicated simulations. The is done separately for each flux component and each histogram bin. 

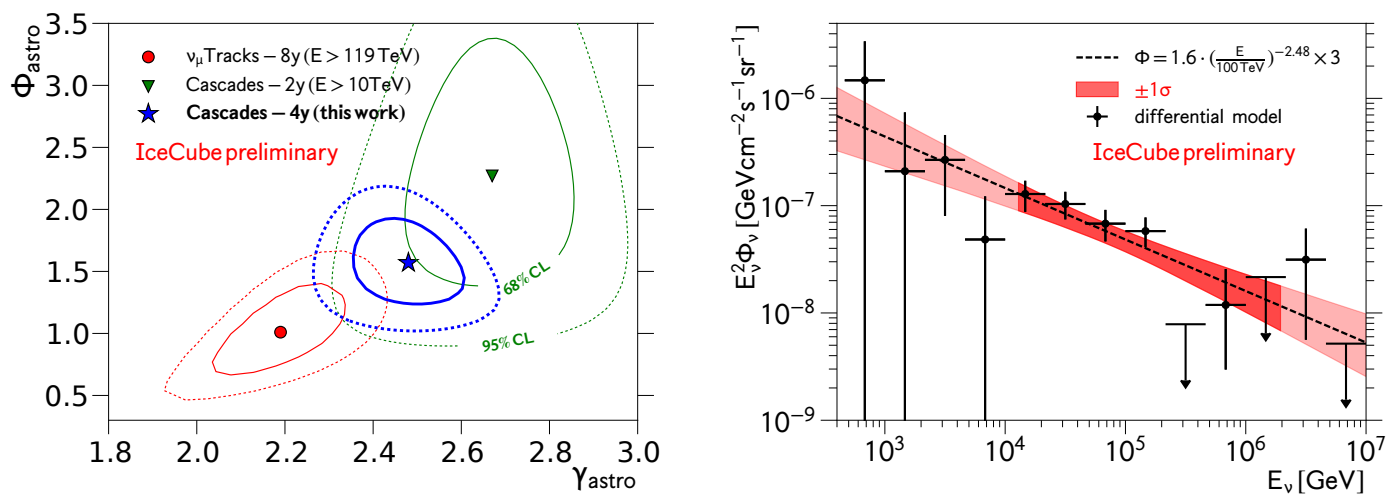

Figure 3: Left: Profile likelihood contours for single power-law parameters; this work (blue), 2-yr cascades (green) and 8-yr diffuse $v_{\mu}$ (red). Right: Best fit of differential flux model (see text), summed over all three $v$-flavors assuming $\left(v_{e}: v_{\mu}: v_{\tau}\right)=(1: 1: 1)$. The sensitive energy range (see text) is highlighted in red.

\begin{tabular}{|llll|}
\hline Parameter & & Prior & Result \\
\hline spectral index & $\gamma$ & - & $\mathbf{2 . 4 8} \pm \mathbf{0 . 0 8}$ \\
norm astro & $\phi$ & - & $\left(\mathbf{1 . 5 7} 7_{-\mathbf{0 . 2 2}}^{+\mathbf{0 . 2 3}}\right)$ c.u. \\
norm conv & $\phi_{\text {conv }}$ & $1.00 \pm 0.30$ & $(1.12 \pm 0.10) \cdot \Phi_{H K K M S 06}$ \\
norm prompt & $\phi_{\text {prompt }}$ & $0.0_{-0.0}^{+1.8} \cdot \Phi_{\text {BERSS }}$ & $<X \cdot \Phi_{B E R S S}\left({ }^{* *}\right)$ \\
norm muon & $\phi_{\text {muon }}$ & - & $1.40 \pm 0.04$ \\
scattering scale & $\varepsilon_{\text {scat }}$ & $1.00 \pm 0.10\left(^{*}\right)$ & $1.07 \pm 0.02$ \\
absorption scale & $\varepsilon_{\text {abs }}$ & $1.00 \pm 0.10\left(^{*}\right)$ & $0.99 \pm 0.03$ \\
dom efficiency & $\varepsilon_{\text {eff }}$ & $0.99 \pm 0.10$ & $1.00 \pm 0.06$ \\
\hline
\end{tabular}

Table 1: Single power-law fit results. (1 c.u. $\left.\equiv 10^{-18} \mathrm{GeV}^{-1} \mathrm{~s}^{-1} \mathrm{sr}^{-1} \mathrm{~cm}^{-2}\right)$. ( $\left.{ }^{*}\right)$ This prior uses a bi-variate normal distribution to account for anti-correlation. $\left(^{* *}\right)$ This upper limit is still under evaluation.

\section{Results}

\subsection{The Single Power-Law}

In this section we assume the astrophysical neutrino flux to follow a single, isotropic, unbroken power-law with equal contributions from all flavors:

$$
\Phi_{v}=\phi \times\left(E_{v} / 100 \mathrm{TeV}\right)^{-\gamma} .
$$

with per-flavor normalization $\phi$ at $\mathrm{E}_{v}=100 \mathrm{TeV}$ and spectral index $\gamma$. The best-fit flux parameters are $\phi=\left(1.57_{-0.22}^{+0.23}\right) \cdot 10^{-18} \mathrm{GeV}^{-1} \mathrm{~s}^{-1} \mathrm{sr}^{-1} \mathrm{~cm}^{-2}$ and $\gamma=2.48 \pm 0.08$. The best-fit values and uncertainties of all other fit parameters can be found in Tab. 1. The flux is measured above the conventional neutrino background in the energy range [7] from $12 \mathrm{TeV}$ to $2.1 \mathrm{PeV}$. Figure 2 shows good agreement between the corresponding reconstructed energy and zenith distributions predicted from our Monte-Carlo simulations and the observed data. The 68\% confidence region (blue) for the astrophysical parameters is shown in Fig. 3 (left) and compared to the result of the previous analysis [9] using 2 years of IceCube data with reconstructed energies larger than $10 \mathrm{TeV}$ (green). Both measurements agree well within uncertainties. Also shown is the result of the most recent IceCube measurement, using muon neutrinos from the Northern Sky, that observed astrophysical neutrinos above $\mathrm{E}_{v}=119 \mathrm{TeV}$ (red) [7]. Both measurements are consistent only at the $\mathrm{p}=0.04$ level as estimated from the two contours. Finally we obtain better constraints on the systematic 
uncertainties compared to the 2-year cascades due to the large number of events at low energies $\left(\mathrm{E}_{\mathrm{rec}}<10 \mathrm{TeV}\right)$, which translates into reduced uncertainties especially in the measurement of the astrophysical normalization.

4.2 The Differential Model While the single power-law appears to be an adequate description of this cascade dataset, we explored a semi-parametric alternative model, the "differential model“", used in previous IceCube analyses [3][8][9]. See ref. [9] for details. The fit result (black) is shown in Fig. 3 (right) and compares well to the parametric single power-law fit (red). Large uncertainties are observed at low energies $\mathrm{E}_{v}<10 \mathrm{TeV}$, where conventional background dominates the spectrum, as well as at highest energies $\mathrm{E}_{V}>200 \mathrm{TeV}$, where only six events were found.

\subsection{The High Energy Tail $(\mathbf{E}>\mathbf{2 0 0 T e V}) \quad$ At energies above $200 \mathrm{TeV}$ we expect essen-} tially no atmospheric background in this sample. Figure 4 (left) shows the likelihood function for a restricted high energy $\mathrm{E}_{\mathrm{rec}}>200 \mathrm{TeV}$ fit with all nuisance parameters fixed to their nominal values. Also shown are the results of previous measurements, the 2-year cascades [9] (black) and the 8-year tracks [7] (white). The six high energy events do not provide sufficient information to distinguish between the two past results and appear consistent with both. The highest energy cascade has a deposited energy of $2 \mathrm{PeV}$ and was reported by IceCube before [3]. We studied the compatibility of this observation with predictions from the 2-year cascades [9] and the 8-year tracks [7], by calculating the predictive distributions (Eq. 3) for the maximum cascade energy to be observed in this sample. The result is shown in Fig. 4 (center). Observing a highest energy cascade with a deposited energy of $2 \mathrm{PeV}$ or less is possible for both assumptions: $\mathrm{P}\left(\mathrm{E}_{\max } \leq 2 \mathrm{PeV} \mid y\right)=0.40$ with $y \equiv 2$-year cascades ( 0.09 with $y \equiv 8$-year tracks). Finally observing only six events or less in total, while interpreted as under-fluctuation, is plausible for both previous results with predicted probabilities of $\mathrm{P}\left(\mathrm{N}_{\text {events }} \leq 6 \mid y\right)=0.14$ with $y \equiv 2$-year cascades $(0.05$ with $y \equiv 8$-year tracks $)$.
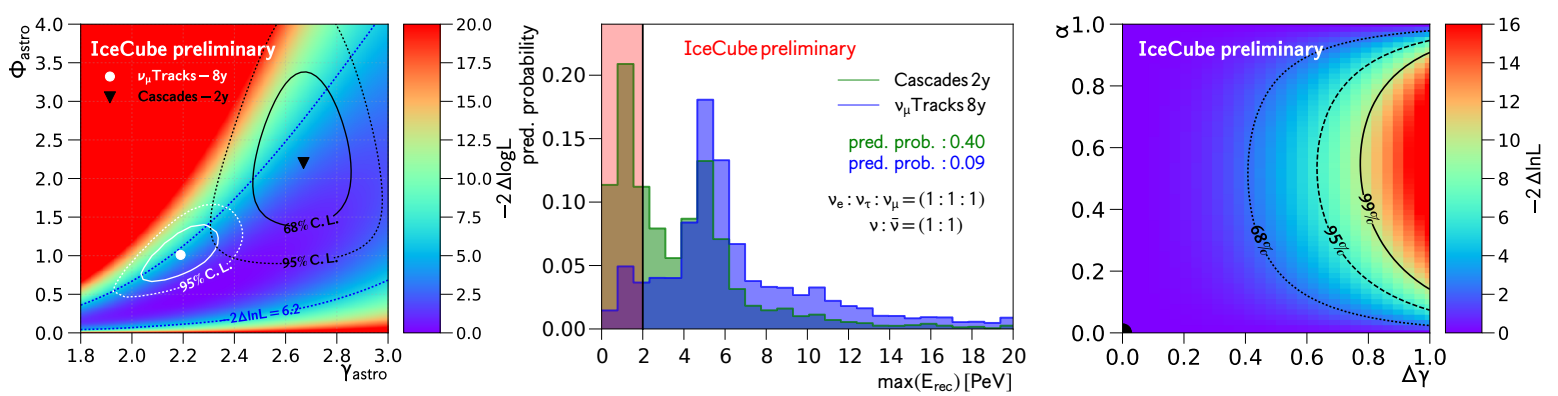

Figure 4: Left: Likelihood function of single power-law model for $\mathrm{E}_{\mathrm{rec}}>200 \mathrm{TeV}$; also shown: 2-year cascades (black) and 8-year diffuse $v_{\mu}$ (white) results. Center: Predicted distribution of maximum observed energy (see text) assuming an unbroken power-law. Right: Profile likelihood scan of parameters describing a possible second power-law component (see text).

\subsection{The 2-Component Power-Law}

Motivated by the 8-year track measurement observing a harder spectrum at energies above $\mathrm{E}_{v}=119 \mathrm{TeV}$ we studied the possibility of a spectral hardening by adding a second power-law component to the astrophysical neutrino flux model:

$$
\Phi\left(E_{v}\right)=\Phi_{0} \times 10^{-18}\left\{(1-\alpha)\left[\frac{E_{v}}{10^{5} \mathrm{GeV}}\right]^{-\gamma_{\mathrm{soft}}}+\alpha\left[\frac{E_{v}}{10^{5} \mathrm{GeV}}\right]^{-\gamma_{\mathrm{soft}}+\Delta \gamma}\right\}
$$


with $0<\alpha<1, \Delta \gamma \geq 0 . \quad \alpha$ denotes the mixture fraction, the fraction of the total flux at $\mathrm{E}_{v}=$ $100 \mathrm{TeV}$ contributed by the harder of the two components. $\Delta \gamma$ describes the amount of spectral hardening. The maximum likelihood best-fit values for the new parameters are $\alpha \rightarrow 0.0$ and $\Delta \gamma=0.0$ such that we find no evidence for the existence of a second component. The fit reduces this model to the single power-law. Figure 5 visualizes the profile-likelihood function for the parameters $\alpha$ and $\Delta \gamma$. The constraints on the parameter $\Delta \gamma$ depend on the mixture fraction $\alpha$. The strongest limits are obtained if both components were to contribute equally to the flux at $\mathrm{E}_{v}=100 \mathrm{TeV}$. In order to interpret this result in the context of the $8 y$-track measurement, we separately calculate HPD regions for the pairs $\gamma_{\text {soft }}, \phi_{\text {soft }}=(1-\alpha) \phi$ and $\gamma_{\text {hard }}=\gamma_{\text {soft }}-\Delta \gamma, \phi_{\text {hard }}=\alpha \cdot \phi$ using Eq. (2), first assuming uniform prior distributions for all four parameters in Eq. (5). Figure 5 (top) shows the resulting contours for the hard (left) and soft (right) components. In this scenario the constraints on the hard component overlap with the 8-year tracks result (red). It is difficult to constrain both components simultaneously using this sample alone due to a strong anti-correlation between the normalization parameters of both components in absence of evidence for spectral hardening in the likelihood. An improved estimate can be obtained by directly interpreting the track result as prior pdf for the hard component, while keeping the identifiability constraint $\gamma_{\text {hard }} \leq \gamma_{\text {soft }}(\Delta \gamma \geq 0)$ as before. The corresponding HPD regions are shown in Fig. 5 (bottom). The posterior pdf for the hard component (blue) shows substantial overlap with the prior distribution (red) and no strong pull
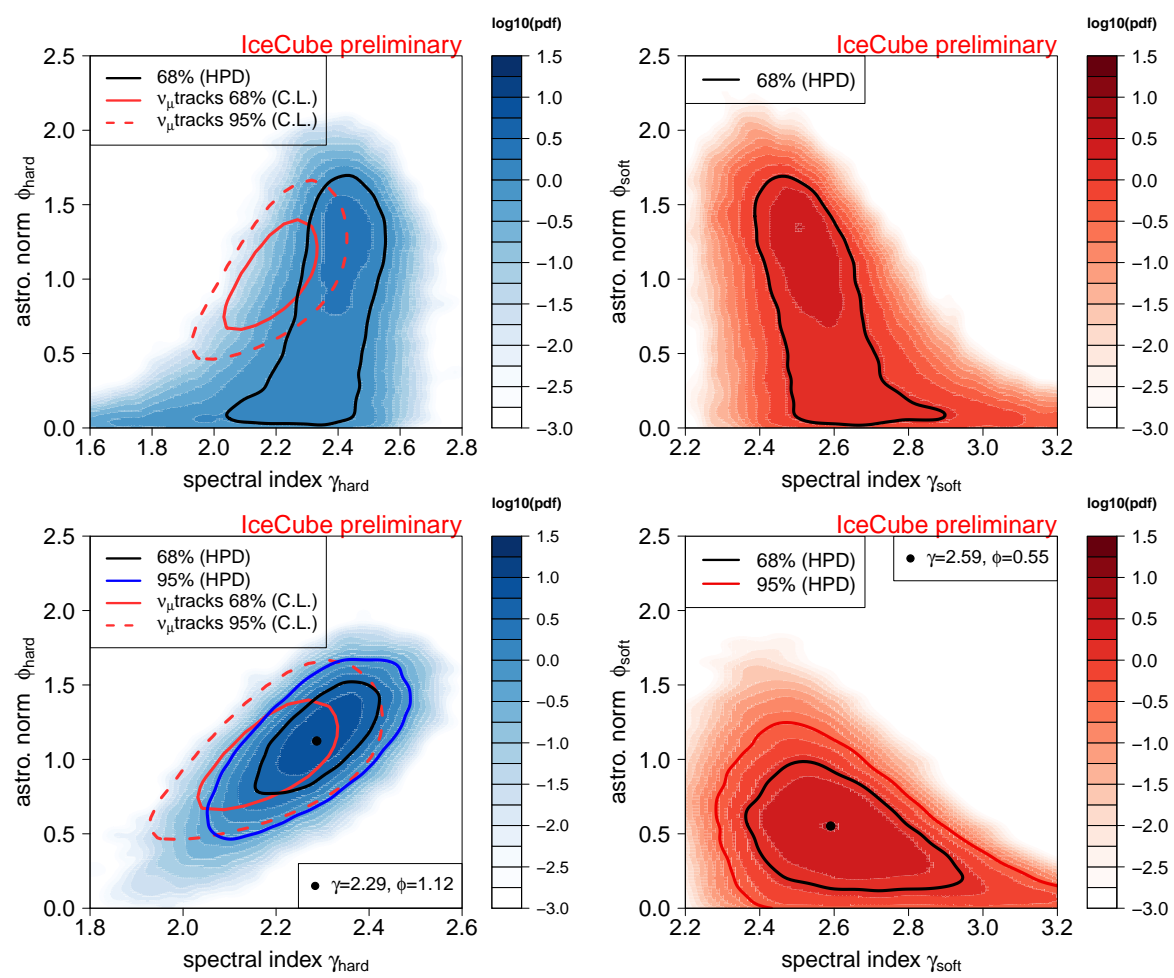

Figure 5: Posterior densities for hard (left) and soft (right) components for different choices of prior distributions (see text): uniform prior distributions (top) and using the result of the 8-year track measurement [7] as prior distribution for the hard component (bottom). Detector related sys. uncertainties are not included. 
is observed. In conclusion, while not preferred, the existence of a harder second flux component with properties given by the 8-year track measurement would be consistent with this dataset.

5. Summary We have performed a new measurement of the high energy astrophysical neutrino flux using neutrino induced cascades observed by IceCube in a four year period (20122015). According to this analysis the astrophysical component is expected to dominate the observed energy spectrum at energies above $20 \mathrm{TeV}$ and appears well described by a single, unbroken power-law. The preliminary fit result is a spectral index of $\gamma=2.48 \pm 0.08$ with a per-flavor normalization at $\mathrm{E}_{v}=100 \mathrm{TeV}$ of $\Phi=\left(1.57_{-0.22}^{+0.23}\right) \cdot 10^{-18} \mathrm{GeV}^{-1} \mathrm{~s}^{-1} \mathrm{sr}^{-1} \mathrm{~cm}^{-2}$ in agreement with previous IceCube analyses. We investigated the possibility of a spectral hardening of the neutrino flux at highest energies as indicated by a recent analysis of muon neutrinos from the Northern Sky by allowing a second power-law component to enter the flux model. While no evidence for the existence of such a component was found, our results do not exclude it either. We further showed that the highest energy events $\left(\mathrm{E}_{\mathrm{rec}}>200 \mathrm{TeV}\right)$ observed in this sample, while being highly informative about the existence of high energy astrophysical neutrinos, due to their small numbers do not provide strong constraints on the spectral behavior of this flux and leave room for interpretation. In the near future we expect improved results from combining this dataset with cascades from the preceding two years as well as from an upcoming fit to all IceCube detection channels [19].

\section{References}

[1] IceCube Collaboration, M. G. Aartsen et al., JINST 12 (2017), P03012

[2] IceCube Collaboration, M. G. Aartsen et al., Science 342, 1242856 (2013)

[3] IceCube Collaboration, M. G. Aartsen et al., Phys. Rev. Lett. 113, 101101 (2014)

[4] T. K. Gaisser, F. Halzen, and T. Stanev, Physics Reports 258, 173 (1995)

[5] E. Waxman and J. Bahcall, Phys. Rev. Lett. 78, 2292 (1997); K. Murase, S. Inoue, and S. Nagataki, Astrophys. J 689, L105 (2008); F. W. Stecker, Phys. Rev. D 88, 047301 (2013)

[6] H. Athar, M. Jezabek and O. Yasuda, Phys. Rev. D 62, 103007 (2000)

[7] IceCube Collaboration, PoS ( ICRC2017) 1005 (these proceedings)

[8] IceCube Collaboration, M. G. Aartsen et al., Phys. Rev. D 91, 022001 (2015)

[9] IceCube Collaboration, PoS ( ICRC2015) 1099 (2015)

[10] D. Heck et al., CORSIKA, Tech. Rep. FZKA 6019, Forschungszentrum Karlsruhe (1998)

[11] M. Honda et al., Phys. Rev. D75, 043006 (2007)

[12] M. G. Aartsen et al., Phys. Rev. D 89, 062007 (2014)

[13] R. Enberg, M. H. Reno and I. Sarcevic, Phys. Rev. D78, 043005 (2008)

[14] A. Bhattacharya et al., JHEP 06, 110 (2015)

[15] IceCube Collaboration, Proceedings of the 33rd ICRC, paper 0370 (2013)

[16] T. Chen and C. Guestrin, Proceedings of the 22nd KDD (2016), arXiv:1603.02754

[17] J. O. Berger, "Statistical Decision Theory and Bayesian Analysis", Springer, 2nd ed. (1993)

[18] A. Gelman et al., "Bayesian Data Analysis", Chapman and Hall/CRC, 3rd ed. (2013)

[19] IceCube Collaboration, PoS ( ICRC2017) 976 (these proceedings) 